\title{
Innovación industrial
}

\section{Industrial innovation}

\section{Rosa Sancho*}

Resumen: Se presentan las definiciones, teorías y modelos del proceso de innovación industrial y su evolución a lo largo del tiempo. Se analiza históricamente el proceso del desarrollo de la innovación en la industria y los indicadores de innovación más comúnmente empleados. Se exponen las encuestas normalizadas que se utilizan en los países industrializados para obtener las estadísticas e indicadores de innovación.

Palabras clave: procesos de innovación, clases de innovación, indicadores de innovación.

\begin{abstract}
A study about the state of the art of definitions, theories and patterns of innovation processes is presented. The process of development of industrial innovation as well as the more commonly used indicators are analysed. The harmonized surveys used in industrialized countries to obtain innovation statistics and indicators are presented.

Keywords: innovation processes, innovation indicators, innovation models.
\end{abstract}

\section{Introducción}

Es un hecho comprobado que el conocimiento y la formación tienen una importancia creciente en el desarrollo económico y social de los países, por lo que las modernas economías se describen como «Economía basada en el conocimiento» y «Economía basada en la formación o aprendizaje» (Knowledge-based Economy y Learning-based Economy), y la sociedad actual se denomina como «Sociedad del conocimiento», lo que refleja claramente la relación de dependencia entre estos conceptos, que se manifiesta en que los sectores industriales más fuertes son aquellos que incorporan decididamente los conocimientos técnicos que se producen, para lo que disponen de recursos humanos altamente cualificados. Dado que la tecnología (conocimiento) se queda obsoleta cada vez más rápidamente, es imprescindible fomentar la formación continuada para producir nueva tecnología. Se podría decir que, actualmente, el principal recurso con que cuenta la economía es el conocimiento y el proceso más importante es el que lleva a dicho conocimiento (aprendizaje).

La habilidad para convertir creativamente el nuevo conocimiento en nuevos o mejores productos y servicios en respuesta a la demanda del mercado y a otras necesidades sociales, es lo que se conoce como innovación, la cual es el origen del cre-

\footnotetext{
* Centro de Información y Documentación Científica (CINDOC-CSIC).
}

Correo-e: rsancho@cindoc.csic.es. 
cimiento económico de los países y de su competitividad internacional e influye positivamente en el nivel de vida de los mismos y en los cambios experimentados por la sociedad $(1,2)$. De tal manera, que el puesto de cada país en el comercio mundial y su renta nacional están ligados a su capacidad de innovación.

\section{Concepto de innovación}

Etimológicamente «innovar» significa producir algo nuevo o introducir mejoras en lo ya conocido. El concepto de innovación va unido a su comercialización, por tanto, se trata de una actividad industrial, y aunque en el sector de la Administración pública (salud, educación, servicios sociales, etc.) se pueden producir innovaciones, estas no se contabilizan y quedan excluidas de las estadísticas de innovación en los países.

En 1992, la OCDE (Organización para la Cooperación y el Desarrollo Económico), consideraba la innovación tecnológica como propia solamente de las industrias manufactureras y enfocada sólo a productos y procesos tecnológicos. La definía como: «la aplicación de una idea nueva hasta su transformación en productos nue vos o mejorados, que se introducen en el mercado, o hasta su transformación en nuevos sistemas de producción, y en su difusión, comercialización y utilización». Entiende también por innovación tecnológica la mejora sustancial de productos o procesos ya existentes (3).

Años más tarde, en 2005, la OCDE amplia el concepto de innovación y la extiende también a las industrias de servicios, siempre que dicha actividad dé lugar a mejoras económicas y mayores ventas en dichas industrias (4). Esta ampliación se justifica por el florecimiento y dinamismo de dicho sector en la mayoría de los países, ya que representa las dos terceras partes de la producción y el $70 \%$ del empleo. Además, dentro de las heterogéneas actividades que abarca (banca, seguros, turismo, consultorías, transporte, informática, etc.), algunas juegan un papel fundamental en la innovación tecnológica, como la industria de «software», p.e. (5)

Además de tener en cuenta el sector servicios, la OCDE amplía el concepto a la innovación no tecnológica y, como resultado, se contemplan también como actividades innovadoras dos nuevos tipos: a) innovación en la organización y gestión de las empresas; y b) innovación en el marketing de las mismas, actividades estas que se consideran también imprescindibles para aumentar su eficacia comercial y productiva.

Entre las actividades de innovación propias de la organización y gestión de las empresas están las relacionadas con la informatización y acceso a redes telemáticas, así como la protección del medio ambiente, la prevención de riesgos, el control de calidad, etc. La innovación en el marketing implica la creación de nuevos métodos de mercadotecnia que supongan cambios significativos en el diseño, promoción, o precio del producto, lo cual se considera también imprescindible para aumentar la eficacia productiva y comercial (6). Ambos tipos de innovación pueden suponer importantes beneficios económicos y sociales para la empresa. 
En todo caso, el requisito exigido para considerar una actividad empresarial como innovativa, es que el producto o proceso, el método de mercadotecnia o el método de organización que se ponga en práctica, sea nuevo o significativamente mejorado para la empresa. Esto incluye tanto los productos, procesos y métodos desarrollados en ellas como los adoptados procedentes del exterior.

Resumiendo, la innovación es un concepto complejo dado su carácter multidisciplinar y ambiguo, ya que designa tanto un proceso como su resultado. Además, tiene un carácter muy amplio, alcanza a las empresas de cualquier tamaño y de cualquier sector industrial, no sólo a las grandes industrias y a los sectores de alta tecnología como biotecnología y tecnologías de la información. Por otra parte, los tipos de innovación pueden variar grandemente, así se puede considerar innovación tanto un avance radical, como puede ser el descubrimiento de un nuevo medicamento o la implantación de cámaras de fotos en los teléfonos móviles, como el diseño de un nuevo tipo de embalaje que ocupe menos espacio, el acceso electrónico a un banco (banco on-line), la mejora en la fabricación o puesta en el mercado de un determinado producto, etc.

\section{Proceso de Innovación}

Inicialmente la innovación se consideraba como el resultado de un proceso lineal de flujo de conocimiento que iría desde la investigación científica básica siguiendo por el desarrollo experimental hasta la fabricación y comercialización del nuevo producto. Este modelo clásico concuerda con la teoría del «impulso creado por la ciencia», según la cual, el desarrollo de ésta conduce a innovaciones que encuentran siempre un lugar en el mercado. Pero se podría adaptar igualmente a la teoría de «la atracción ejercida por la demanda», ya que la demanda de un producto por los consumidores impulsa la actividad creativa e innovadora (7).

Según este modelo, sería suficiente concentrar todos los esfuerzos en los primeros estadios para obtener los resultados esperados, lo que ha sido la justificación para considerar los gastos en I+D como el principal indicador de la actividad de innovación.

Sin embargo, se observó que los procesos que dan lugar a la innovación se ajustan mejor a un modelo interactivo y multidimensional (8), según el cual la actividad de innovación es el resultado de un complejo proceso de interacciones, a veces durante un largo periodo de tiempo, entre diferentes actividades heterogéneas e interdependientes, tales como: investigación y desarrollo, ingeniería, estudio de mercados y de usuarios, planificación financiera, canales de distribución, características de los proveedores de equipos y materias primas, opiniones de los clientes, etc. En definitiva, se trata de un proceso que mantiene fuertes enlaces entre la ciencia, la tecnología, los consumidores y el mercado.

Según este modelo interactivo, la I+D ha dejado de ocupar el primer puesto en el proceso de innovación industrial, sobre todo en el sector de los servicios, y su importancia varía grandemente según los diferentes sectores industriales. Por ejem- 
plo, en el sector farmacéutico y biotecnológico la investigación básica que tiene lugar principalmente en las universidades es crucial para lograr la innovación técnica de las industrias. La correlación entre la I+D y la innovación es aquí muy alta. Sin embargo, en otros sectores, como alimentación, tejidos, maquinaria, etc., sólo un pequeño porcentaje de investigación básica se convierte en nueva tecnología, y usualmente con gran retraso.

En el sector de los servicios el éxito en la innovación depende principalmente de factores ajenos a la I+D, como son la tecnología adquirida, la calidad de los recursos humanos y la organización de la empresa. Precisamente, algunos autores consideran que la clave para conseguir innovaciones en las empresas de dicho sector se basa en el análisis de las necesidades del mercado y de los consumidores para poder adaptar sus productos o métodos de producción a dichas necesidades $(9,10)$.

Es también el caso de las PYME que, con poco o nulo nivel de I+D, logran llevar a término innovaciones con éxito, gracias al contacto directo con los clientes o utilizando tecnologías ya desarrolladas para hacer más eficiente su producción, o empleando nuevas técnicas para la organización y el marketing, actividades estas fundamentales para su supervivencia y competitividad.

En general, la disponibilidad de recursos humanos cualificados y de una infraestructura de comunicaciones bien desarrollada, entre otros, tiene mucha importancia en el proceso innovativo (11). Se demuestra que una buena gestión de los recursos humanos de la empresa influye positivamente en la innovación, lo que incluye la creación de equipos de trabajo multidisciplinares formados por expertos de varias secciones de la misma, rotación en el trabajo, formación continuada, etc. (12)

\section{Clases de Innovación Industrial}

Siguiendo las definiciones de la OCDE, se describen a continuación las cuatro categorías en que se divide la innovación industrial, las dos primeras se pueden considerar innovación técnica, y las dos siguientes de gestión (4):

- Innovación de productos, que se considera «realizada» sólo cuando se introducen en el mercado tanto los productos enteramente nuevos (innovación radi cal), como los productos ya existentes, pero sustancialmente mejorados (inno vación incremental). Esto incluye las mejoras significativas en las especificaciones técnicas, componentes o materiales, «software» incorporado, etc. Las innovaciones incrementales provienen mayoritariamente del trabajo de ingenieros y técnicos y de los estudios de mercados, mientras que las radicales se basan, principalmente en la ciencia.

- Innovación de métodos o procesos de producción, que supone la introducción en el mercado tanto de nuevos procesos o métodos de producción como de procesos ya existentes, pero mejorados. Incluye cambios significativos en las técnicas, en los equipos empleados, en «software», etc. 
- Innovación en la gestión, que significa el cambio o mejora en la organización o en la gestión e la empresa, incluyendo nuevos métodos en la práctica de la organización del trabajo o en las relaciones externas.

- Innovación en el marketing, que comprende la creación de nuevas estructuras de mercadotecnia en la empresa, incluyendo cambios en el diseño o empaquetado del producto, en su promoción, o en el precio.

De lo dicho anteriormente se deduce que la innovación no siempre es una invención, pero, siempre implica novedad, y ésta puede ser considerada novedad en el mundo (máxima innovación), o sólo en un país, o incluso únicamente en la empresa determinada (mínima innovación). A las empresas que ponen en práctica los desarrollos iniciados en otras, se conocen como imitadoras. Las innovaciones incrementales y las imitaciones de productos o procesos ya existentes y su adaptación a los mercados, son, como se ha dicho, los tipos de innovaciones más desarrollados en las PYME.

\section{Desarrollo de la Innovación en la Industria}

La evolución de las necesidades de los consumidores, el desarrollo de nuevas tecnologías, y la liberalización del comercio internacional, entre otros, han originado en las últimas décadas profundos cambios en la estructura, estrategia, actividades y funcionamiento de las industrias. Por otra parte, la globalización ha aumentado notablemente el acceso a la información y ha abierto nuevos mercados, lo que ha llevado a una mayor competitividad internacional.

La presión para reducir los precios crece continuamente mientras aumentan las exigencias en las normas de calidad. Bajo estas condiciones, el único sistema para hacer frente a la competencia industrial es el desarrollo de productos y procesos innovadores. Actualmente, las empresas más competitivas son las que tienen mayor capacidad de innovación.

La creación de nuevos productos o procedimientos para asegurar la supervivencia de la empresa se ve estimulada no sólo por la competencia entre las mismas, son también por la aparición de mercados en decadencia (caída de las ventas). Por otra parte, la compra y adquisición de tecnología externa, incorporada en equipos o maquinaria, facilita la innovación basada directamente en dicha tecnología externa adquirida e influye positivamente en la competitividad industrial, pero hace disminuir el desarrollo de la propia innovación interna.

Este es el caso de España, donde el tejido empresarial está constituido principalmente por empresas de pequeño tamaño y pocos recursos (PYME) y donde la innovación es inducida principalmente por los proveedores de equipos y materias primas, y donde la demanda del público como impulsor de la innovación es menos activa que en otros países. Además, en las empresas españolas, la mayoría de las innovaciones consisten en mejoras graduales de productos y procesos ya existentes, 
debido, principalmente, a la falta de financiación capital-riesgo privado y a una débil participación de compañías extranjeras, a una industria de baja intensidad en I+D, especialmente la industria de fabricación, así como a una escasa o nula relación entre el sector académico y el productivo (13).

Entre otros factores externos a la empresa que contribuyen al éxito de la innovación, se pueden señalar las ayudas financieras del estado (reducciones de impuestos, concesión de créditos blandos, incentivos fiscales etc.), y el establecimiento de colaboraciones y contratos con organismos públicos de investigación o universidades, tanto para la utilización común de equipos costosos, realización de proyectos de I+D conjuntos, etc., como para facilitar el intercambio y movilidad de recursos humanos (ingenieros, técnicos y científicos).

\section{Indicadores de Innovación Industrial}

Es necesario disponer de indicadores que reflejen las actividades de innovación en las industrias, e indiquen los factores que influyen o dificultan su capacidad innovativa. Como se ha dicho, durante mucho tiempo se han considerado los gastos en $\mathrm{I}+\mathrm{D}$ como indicadores de inversiones de los procesos innovadores y las solicitudes y concesiones de patentes como medida directa de los resultados, pero se ha demostrado que éstos proporcionan sólo una medida parcial de la innovación, porque concentran la atención en un grupo muy restringido de industrias y sus actividades.

Por tanto, es necesario generar nuevos indicadores que tengan en cuenta la dinámica de la innovación en un contexto social y económico más amplio. Por otra parte, el seguimiento del proceso innovativo se dificulta porque suele transcurrir un largo periodo de tiempo hasta que se consigue una innovación de importancia económica y su difusión en el mercado (14).

Entre los años 1980 y 1990, aproximadamente, los datos nacionales sobre las actividades de innovación se recopilaban en los Institutos Nacionales de Estadística, por medio de encuestas enviadas a las industrias de cada país, las cuales, aunque similares, no eran homogéneas y no podían compararse entre sí.

Debido a la ausencia de datos sistemáticos fiables, en 1990 la OCDE, acordó que: «Es necesario que los países miembros colaboren en el desarrollo de nuevos indicadores de innovación, normalizados, que sean comparables internacionalmen te y que midan con precisión la compleja actividad de innovación y, sobre todo, sus interacciones con la economía» (15).

Para conseguir dicho propósito, la OCDE publicó en 1992 las primeras directrices para el recuento e interpretación de datos de innovación tecnológica (Manual de Innovación, conocido como Manual de Oslo) (3). Dicha publicación forma parte de una familia de Manuales, creados por dicha institución y dedicados a la obtención de indicadores relacionados con la ciencia, la tecnología y la innovación. El Manual de Oslo proporciona la metodología para diseñar las encuestas dirigidas a las empresas, de una manera normalizada en todos los países, para que los datos estadísticos 
obtenidos sean comparables internacionalmente y proporcionen indicadores sobre el proceso de innovación y el impacto de ésta en la economía, así como los obstáculos para conseguir dicho proceso.

Para ello, se envía a las empresas una encuesta armonizada común (Community Innovation Survey, CIS) (16), fruto de un proyecto de colaboración entre la OCDE y EUROSTAT (Oficina de Estadísticas de la Unión Europea), y cuyos cuestionarios siguen las directrices del Manual de Oslo. En 1992 se utilizó por primera vez el CIS 1, en 1997 el CIS 2, con contenidos más depurados y teniendo en cuenta los diferentes sectores de la economía (industrias de fabricación, servicios, agricultura, construcción, etc.). Actualmente se ha concluido el análisis del CIS 4, que contempla con más extensión aspectos no técnicos de la innovación, tales como procedimientos de gestión, cambios en la organización y marketing.

\section{Indicadores de inversiones. Encuesta CIS}

Los cuestionarios CIS permiten recuperar información según las principales variables siguientes.

- $\mathrm{N}^{\mathrm{o}}$ empresas innovadoras.

- Tipo de innovación que realizan.

- Países u organizaciones con quien cooperan las empresas (empresas del mismo grupo, universidades, OPI, clientes, proveedores, consultoras).

- $\mathrm{N}^{\circ}$ empresas que han recibido financiación pública para la innovación (Estado, CCAA, UE).

- $\mathrm{N}^{\mathrm{o}}$ de empresas que adquieren equipos, maquinaria y «software».

- $\mathrm{N}^{\mathrm{o}}$ empresas que realizan I+D (sistemática, ocasional).

$-\mathrm{N}^{\mathrm{o}}$ patentes registradas.

- $\mathrm{N}^{\mathrm{o}}$ empresas con innovaciones no tecnológicas (cambios estrategia corporativa o de marketing, estética, diseño, reducción de costes o energía por unidad de producción, reducción impacto ambiental, etc.).

- Obstáculos para la innovación (riesgos económicos, elevado coste, falta de financiación, falta de personal cualificado, poca flexibilidad en la empresa, falta de información sobre mercados potenciales o sobre tecnología, etc.).

- Gastos totales en innovación.

- Gastos en innovación por CCAA.

- Porcentaje de la cifra de negocios dedicada a innovación.

-Intensidad de innovación (gastos en innovación/ cifra de negocio).

En España, el INE (Instituto Nacional de Estadística) (17) publica cada dos años los resultados de la encuesta de innovación CIS que envía a las empresas de más de 10 trabajadores (18). Quedan así recogidos los datos estadísticos sobre inversiones en innovación, y sobre facilidades y obstáculos a la misma. 
Según dichos datos, el porcentaje de empresas españolas que hacen innovación ha aumentado desde 1998, cuando el 10\% del total de empresas obtenía resultados innovadores, hasta 2005 , último dato suministrado, con un $27 \%$ del total de empresas. En el año 2004, en el que España presenta un 29,7\% de empresas innovadoras, la media europea de empresas innovadoras se situaba en un 44\%; Alemania era el país con mayor proporción de ellas, llegando al 65\%, y Francia, contaba con el $32 \%$ (19). Asimismo, en España crece anualmente el gasto en innovación, desde 130.000 Mpts en 1998, hasta 13.636 M € en 2005. La variación menos evidente es el porcentaje sobre la intensidad de innovación, que se mide por el gasto de innovación en relación con la cifra de negocio, y que se mantiene sin grandes variaciones a lo largo del tiempo alrededor del $2 \%$ (17).

Por ramas de actividad, las empresas de fabricación de vehículos de motor representan el mayor porcentaje del gasto industrial total en innovación, con un rango entre $17 \%$ y $12 \%$ del gasto total en innovación, en el periodo 1998-2005, le siguen las empresas de alimentos y bebidas (11\% y 9\%, del gasto total en el mismo periodo). En el sector de los servicios, como informática y programas de ordenador, se gasta alrededor del $40 \%$, manteniéndose anualmente dicho porcentaje durante el periodo 2003-2005 (17).

En relación al porcentaje de empresas innovadoras respecto al total de empresas por sector, figura en primer lugar el sector del petróleo, ya que el $80 \%$ de todas sus empresas se reconocen como innovadoras en el año 2003, le sigue el sector de la aeronáutica, con el $71 \%$ de todas sus empresas innovadoras, en el año 2005. En ese mismo año, el sector de radio y televisión llegaba al $71 \%$ de empresas innovadoras, y en el de servicios, tanto financieros como de programas de ordenador, más de la mitad de sus empresas se declaran innovadoras en dicho año (17).

\section{Indicadores de resultados de la innovación}

Estos indicadores tienen bastantes limitaciones. En general se obtienen de fuentes externas a las empresas y no se contemplan en las encuestas CIS. Los utilizados más frecuentemente son los basados en estadísticas de patentes $\left(\mathrm{n}^{\circ}\right.$ patentes solicitadas y concedidas, $\mathrm{n}^{\mathrm{o}}$ patentes en el extranjero, etc.), asumiendo que éstas reflejan la actividad de innovación (20). Sin embargo, las patentes son invenciones y, como se ha dicho, las innovaciones raramente lo son, por tanto, muchas de las innovaciones no pueden ser patentadas, sobre todo las relacionadas con gestión y marketing. Además, las patentes informan de la fase de invención, pero casi nunca de su comercialización, y, por tanto, de su impacto económico (21).

En España, los indicadores de patentes solicitadas y concedidas en el sector empresas ofrecen cifras muy bajas en comparación con otros países de la OCDE. Así en el año 2002, se contabilizaron en España 25 patentes solicitadas a EPO (European Patent Office) por millón de habitantes, frente a 349 de Suiza, 253 de Alemania, 226 de Finlandia, etc. (22). Para facilitar la comparación internacional y 
eliminar sesgos, la OCDE ha desarrollado indicadores de familias de patentes triádicas, que son las solicitadas simultáneamente a las oficinas de patentes de Japón, EEUU y Europa, para proteger la misma invención. España, en 2001, contaba con 3 patentes triádicas por millón de habitantes, mientras Suiza alcanzaba la cifra de 120, seguida de Finlandia, con 100 (23).

La Transferencia de Tecnología en las Empresas se mide por los datos extraídos de la Balanza Tecnológica de Pagos (BTP), que mide la competencia internacional de un país a través del impacto económico que produce la compra y venta de tecnología en el extranjero, en relación con los derechos de propiedad industrial (patentes, licencias, «know-how», asistencia y servicios técnicos de asesoría, de informática, etc.). Sin embargo, ofrece una visión parcial del fenómeno general de transferencia de tecnología ya que contempla sólo la difusión internacional de ésta.

El déficit de la BTP se traduce en un grado de dependencia tecnológica del país con respecto al exterior. En el caso de España, se registra permanentemente un saldo muy negativo, en 1995 (últimos datos disponibles), recibía 79 millones de dólares y pagaba 1.110 , lo que da idea del alto grado de dependencia tecnológica del exterior. (23).

Cabría citar también los indicadores relacionados con procesos económicos que tienen en cuenta las ventas y exportaciones de nuevos productos.

Entre otros indicadores de resultados se pueden mencionar los que miden las innovaciones de productos que han sido comercializados y se publican en las revistas técnicas de ingeniería, en la sección de anuncios de nuevos productos, que en ningún caso se considera propaganda publicitaria (indicador de resultados de innovación basado en literatura: «Literature-based innovation output indicator», LBIO). Las listas de algunas de dichas revistas se ofrecen en (24) y (25). Estos indicadores ignoran las mejoras incrementales y no tienen en cuenta las innovaciones de proceso que no aparecen publicadas, porque, generalmente, se suelen mantener en secreto.

Los indicadores que miden y evalúan las mejoras en la innovación tecnológica son los llamados indicadores tecnométricos que analizan los cambios técnicos en los productos de innovación basados en la comparación de las características técnicas de los mismos y la calidad de sus especificaciones (26).

\section{Conclusiones}

La innovación no es un fenómeno que se produce aisladamente en una empresa, sino que para que se realice con éxito tiene que existir un contexto favorable de relaciones estructurales con otras empresas, instituciones públicas, clientes, proveedores, etc. Por tanto, en este escenario juegan un papel predominante los Sistemas Nacionales de Innovación, constituidos por instituciones tanto del sector público (centros de investigación, universidades, administración del estado, etc.), como del sector de mercado (empresas privadas), cuyas interacciones favorecen los mecanismos de transferencia de conocimiento, lo que es fundamental en los procesos de 
innovación. La vitalidad de los SNI depende del número y calidad de las diferentes instituciones que conforman el sistema y de la eficaz cooperación que se establezca entre ellas. El hecho de que la innovación sea un proceso acumulativo e interactivo significa que la habilidad para innovar refleja las buenas relaciones y la interconexión que existe entre las instituciones.

Los SNI se enmarcan en el modelo de la Triple Hélice («Triple Helix»), que refiere las complejas interrelaciones que se producen en la red universidad-industria-gobierno, cuyo dinamismo conduce a la formación de interfaces entre los componentes de la red y a transformaciones internas dentro de ellos. Así, por ejemplo, la universidad ha pasado de ser una institución de enseñanza a combinar ésta con la investigación, lo que favorece la interacción con la industria y con la sociedad, en un proceso de intercambio de conocimiento $(27,28)$

El concepto de globalización ha variado la perspectiva de los SNI, que fueron concebidos con la idea de que muchos de los factores que influyen en las actividades de innovación eran de carácter nacional (instituciones, cultura, etc.), sin embargo, en la actualidad los procesos de innovación son, en muchos casos, de carácter internacional (29). Las empresas interaccionan con otras empresas o universidades extranjeras, Internet permite la libre circulación de ideas, además de comunicarse y hacer negocios con cualquier país, y se observa que la aplicación de las tecnologías fuera de las fronteras de un país está muy generalizada. Por lo general, en las empresas multinacionales las tecnologías novedosas todavía se desarrollan en la empresa principal, pero existe una tendencia creciente hacia la localización de otras actividades de innovación en las filiales situadas en países extranjeros (30).

Las actuales políticas de innovación tienden a dirigir la investigación básica, que ordinariamente se produce en las universidades, hacia una investigación más orientada a las necesidades de mercado, debido, precisamente, a la creciente presión del sector privado. En este sentido, en algunos países el contexto de la investigación en las universidades está cambiando favorablemente, ya que éstas se ven obligadas a trabajar en cooperación con las industrias, e incluso pueden formar parte de empresas conjuntas («joint ventures», «spin-offs»). Sin embargo, en la mayoría de los países, las universidades y los centros públicos de investigación todavía contribuyen sólo de forma marginal al desarrollo de la innovación comercial.

Para que las actividades de transferencia de tecnología del sector público al privado sean eficaces, se requiere la creación de interfaces entre ambos sectores que faciliten la comunicación entre ellos. Las llamadas «ciencias de transferencia» (ciertas ramas e la ingeniería, tecnologías de la información, de materiales, etc.), situadas entre las ciencias puras y el sector productivo, juegan un papel esencial en el establecimiento de relaciones fructíferas entre ciencia e industria.

No hay que olvidar que la política de innovación tiene objetivos más amplios que los de la política científica y tecnológica, e incluye también las políticas de cambios en la organización de la empresa y en el marketing de nuevos productos, aspectos éstos de consecuencias socioeconómicas importantes, y que, a menudo, se ignoran en las políticas de fomento a la innovación. 
Teniendo en cuenta que todo proceso de innovación lleva consigo una serie de actividades, con frecuencia de alto riesgo, como son los gastos económicos excesivos, la falta de fuentes apropiadas de financiación, la rigidez en la organización de la empresa, la falta de personal cualificado, la falta de sensibilidad a los nuevos productos, etc., es necesario que la política industrial de los países contemple la asignación de recursos públicos para fomentar y reforzar dichas actividades relacionadas con la innovación, aplicando políticas de incentivos fiscales y créditos.

\section{Bibliografía}

1. SCHUMPETER, J. A. (1934). The theory of Economic Development. Harvard University Press. Cambridge. Mass.

2. ROSENBERG, N.; LANDAU, R.; MOWERY, D. (1992). Technology and the Wealth of Nations. Introduction. Rosenberg, Landau and Mowery (eds). Stanford University Press. Stanford CA.

3. OCDE. (1991) Proposed guidelines for collecting and interpreting technological innovation data. Oslo Manual. OCDE. París, 1991.

4. OCDE. (2005). Proposed guidelines for collecting and interpreting technological innovation data. Oslo Manual. ( $3^{\circ}$ edition). OECD. París, 2005.

5. MILER, I (2005). Innovation in Services. Chapter 16, in J. Fagerberg, D. Mowery and RR Nelson (eds). The Oxford Handbook of Innovation. Oxford University Press, Oxford

6. LAM, A., (2005) Organizational Innovation. Chapter 5 in J. Fagerberg, D. Mowery and RR Nelson (eds). The Oxford Handbook of Innovation. Oxford University Press, Oxford

7. SCHMOOKLER, J. (1966). Invention and Economic Growth. Cambridge (Mass). Harvard University Press.

8. KLINE, S.J.; ROSENBERG, N.(1986). An overview of innovation. En: Landan, R.; Rosenberg, N. (Editors). The Positive Sum Strategy. Harnessing Technology for Economic Growth. Washington. D.C. National Academic Press, p. 275-306, 1986.

9. GOBELI, D. H.; BROWN, D. J. (1993). Improving the process of product innovation. Research Technology Management, v. 36, $\mathrm{n}^{\circ}$ 2, pp. 38-44.

10. FREEMAN, C. (1994). The economics of technical change. Cambridge Journal of Eco nomics. v. $18, \mathrm{n}^{\circ} 5$, pp.463-514.

11. MOEN, J. (2005). Is mobility of technical personnel a source of R\&D spillovers?. Jour nal of Labour Economics. 23 (1) p. 81-114.

12. LAURSEN, K., FOSS, N. (2000) New HRM practices, complementarities and the inpact of innovation performance. IVS Working Paper, $\mathrm{n}^{\circ} 5$. Copenhagen Business School.

13. FUNDACIÓN COTEC PARA LA INNOVACIÓN TECNOLÓGICA.(2006). Tecnología e innovación en España.. Informe COTEC, ISBN: 84-95336-66-9. 2006.

14. EUROPEAN COMMISSION. (1996). International Conference. Innovation Measurement and Policies. Luxembourg, 20-21 May, 1996.

15. OECD (1990). Description of Innovation Surveys and Surveys of Technology use carried out in OECD member countries. OECD. París.

16. COMISIÓN EUROPEA. (1996). Encuesta Comunitaria sobre Innovación. 1992. Bruselas. 
17. INE. www.ine.es/inebase

18. INE (Instituto Nacional de Estadística).(1991). Encuesta sobre Innovación Tecnológica en las Empresas. Estudio piloto.

19. www.mityc.es/es-ES/Servicios/Indicadores

20. ARCHIBUGI, D. (1992). Patenting as an indicator of technological innovation. A review. Science and Public Policy. v. 19, ${ }^{\circ}$ 5, p. 1-13

21. PAVITT, K. (1982). R\&D patenting and innovative activities. A statistical exploration. Research Policy, v. 11, no 1, p. 33-51, 1982.

22. OECD (2005). Science and Technology and Industry Scoreboard. Towards a Knowledge based Economy.2005.

23. OCDE (2006) Main Science and Technology Indicators, vol 2006/2

24. COOMBS, R; NARANDREN, P.; RICHARDS, A. (1991). A literature-based innovation output indicators. Research Policy, n ${ }^{\circ} 25$, p. 403-413.

25. SANTARELLI. E; PIERGIOVANNI, R. (1996). Analyzing literature-based innovation output indicators. The Italian experience. Research Policy, $\mathrm{n}^{\circ} 25$, p. 689-711.

26. FRENKEL, A., MAITAL, S., GRUPP, H. (2000). Measuring dynamic technological change: a technometrics approach. International Journal of Technology Management, vol. $20, \mathrm{n}^{\circ} 3 / 4$, p. 429-441.

27. ETZKOWITZ, H. LEYDESDORFF, L. (2000). The dinamics of innovation: from National Systems and mode 2 to a Triple Helix of university-industry-government relations. Research Policy, vol.29, p. 109-123.

28. LEYDESDORFF, L, MEYER, M. (2006) Triple helix indicators of knowledge-based innovation systems. Introduction to the special issue. Research Policy, vol. 35, p. 14411449.

29. LUNDVALL, B. A. (2002). Innovation Growth and Social Cohesion. The Danish model. Edward Elgar Publishing, Inc. ISBN 183064743 4. p. 219.

30. ARCHIBUGUI, D.; MICHIE, J. (1995). The globalization of Technology: a new taxonomy. Cambridge Journal of Economy, 19 (1), p. 121-140. 